\title{
Association of TAP Gene Polymorphisms and Risk of Cervical Intraepithelial Neoplasia
}

\author{
Camilla Natter, ${ }^{1}$ Stephan Polterauer, ${ }^{1}$ Jasmin Rahhal-Schupp, ${ }^{1}$ Dan Cacsire Castillo-Tong, \\ Sophie Pils, ${ }^{1}$ Paul Speiser, ${ }^{1}$ Robert Zeillinger, ${ }^{1}$ Georg Heinze, ${ }^{2}$ and Christoph Grimm ${ }^{1}$ \\ ${ }^{1}$ Department of General Gynaecology and Gynaecological Oncology, Comprehensive Cancer Center, Medical University of Vienna, \\ Waehringer Guertel 18-20, 1090 Vienna, Austria \\ ${ }^{2}$ Center for Medical Statistics, Informatics and Intelligent Systems, Section for Clinical Biometrics, Medical University of Vienna, \\ 1090 Vienna, Austria
}

Correspondence should be addressed to Camilla Natter; camilla.natter@meduniwien.ac.at

Received 5 December 2012; Accepted 2 May 2013

Academic Editor: Sudhir Srivastava

Copyright (C) 2013 Camilla Natter et al. This is an open access article distributed under the Creative Commons Attribution License, which permits unrestricted use, distribution, and reproduction in any medium, provided the original work is properly cited.

\begin{abstract}
Background. Transporter associated with antigen processing (TAP) is responsible for peptide loading onto class I major histocompatibility complex (MHC-I) molecules. TAP seems to facilitate the detection of HPV by MHC-I molecules and contributes to successful eradication of HPV. TAP polymorphisms could have an important impact on the course of HPV infection. Objective. The aim of this study is to evaluate the association between five TAP gene polymorphisms and the risk of CIN. Methods. This case-control study investigated five common TAP polymorphisms in TAP1 (1341 and 2254) and TAP2 (1135, 1693, and 1993) in 616 women with CIN and 206 controls. Associations between gene polymorphisms and risk of CIN were analysed by univariate and multivariable models. The combined effect of the five TAP gene polymorphisms on the risk for CIN was investigated by haplotype analysis. Results. No significant difference in genotype distribution of the five TAP polymorphisms was observed in women with $\mathrm{CIN}$ and controls. Haplotype analysis revealed that women with haplotype mut-wt-wt-wt-wt (TAP polymorphisms t1135-t1341t1693-t1993-t2254) had a significantly lower risk for CIN, compared to women with the haplotype wt-wt-wt-wt-wt $(P=0.006$; OR 0.5 [0.35-0.84]). Conclusion. Identification of this haplotype combination could be used to identify women, less susceptible for development of CIN following HPV infection.
\end{abstract}

\section{Introduction}

Cervical intraepithelial neoplasia (CIN) is a premalignant disease, often leading to invasive cervical cancer [1]. Cervical cancer is the second most common cancer in women [2]. It is widely known that CIN is caused by an infection with highrisk human papillomavirus (HPV) types [3, 4]. Interestingly, most HPV infections are transient and do not lead to CIN [5].

Genetic, immunologic, and socioeconomic factors play a role in the natural history of HPV infection and development of cervical intraepithelial neoplasia. Lifestyle factors, such as cigarette smoking, are well known to be a risk factor for HPV persistence and development of cervical intraepithelial neoplasia, the precursor lesion of cervical cancer [6]. Compared to women who have never smoked, current cigarette smokers have a significantly increased risk of high-grade cervical intraepithelial neoplasia and cervical cancer [7].

Host factors such as the immune systems and genetic factors also seem to be important factors in HPV persistence and subsequent cervical cancer carcinogenesis $[8,9]$.

The transporter associated with antigen processing (TAP) belongs to the superfamily of ATP-binding cassette (ABC) transporter that is essential for peptide loading onto class I major histocompatibility complex (MHC-I) molecules [10, 11]. TAP genes are encoded in the MHC class II region of chromosome 6 . Transporter associated with antigen processing is composed of two integral membrane proteins, TAP1 and TAP2, which have one hydrophobic region and one ATP-binding region each. They assemble into a heterodimer, which results in a four-domain transporter. TAP 1 functions 
by providing a supply of candidate peptides to the MHC I molecules within the peptide loading complex and by transporting antigen peptides from the cytoplasm into the endoplasmic reticulum (ER) $[12,13]$.

MHC-I molecules play a major role in the immune response against viral infections and transformed cells by presenting peptide antigens to cytotoxic $\mathrm{T}$ lymphocytes (CTL) $[14,15]$. The loss of MHC class I expression is observed commonly in various tumours and is possibly the mechanism underlying the ability of neoplastic cells to evade the immune system [16]. Regarding cervical cancer, MHCI antigen has been reported to be downregulated in HPV-16 and -18 positive cervical malignancies [17]. TAP facilitates the detection of HPV by MHC-I molecules and contributes to successful detection and eradication of HPV despite various immunoevasion mechanisms of the virus [18]. Therefore TAP gene polymorphisms have been investigated in women with cervical cancer precursor lesions and in women with cervical cancer [19-22]. Einstein et al. ascertained a reduced risk for high-grade CIN at the presence of TAP1 I333V and TAP1 D637G.

In the present study, we evaluated the association between five common TAP gene polymorphisms in TAP1 and TAP2: TAP1 1341 (rs1057141), TAP1 2254 (rs1135216), TAP2 1135 (rs1800454), TAP2 1693 (rs2228396), and TAP2 1993 (rs241447) and the risk of CIN in a total number of 822 women.

\section{Materials and Methods}

Women, who were referred to the Department's outpatient clinic for genital dysplasia between 2004 and 2009 because of a cytological result "atypical cells of undetermined significance (ASC-UCS)" or higher, were asked to participate at the present study. In total, 616 Caucasian women with histologically proven CIN 1-3, treated at the Department of Gynaecology and Gynaecological Oncology, Medical University of Vienna, Comprehensive Cancer Centre, Vienna, Austria, were included in this study.

The examination in our outpatient clinic comprised ectoand endocervical cytology, human papilloma virus (HPV) DNA testing (Hybrid Capture 2-test, Digene Corporation, Gaithersburg, MD, USA), application of 3\% acetic acid, colposcopy, and colposcopically guided biopsy. An additional cervical smear was taken to collect the DNA sample. After the women agreed to participate at the study by signing informed consent, they were asked about their smoking status (yes including previous smokers versus no), and the clinical examination was performed. The patients, in whom no CIN 1-3 was diagnosed after colposcopically guided biopsy, were excluded of further analysis ( $n=60$ patients).

As a control group, we included 206 consecutive Caucasian women, who presented at the Department's gynaecologic outpatient clinic between 2004 and 2009 with vaginal infection, pelvic pain or similar symptoms, and without a history of conization or histologically proven CIN 1-3. Patients, who agreed to participate, signed informed consent and had in addition to their particular examination, ecto- and endocervical cytology and one cervical smear to collect the DNA samples. Only patients with normal cytology were included in the control group. No HPV-testing was done for patients in the control group.

Patients with known HIV, hepatitis B or C infection, or a history of gynaecologic malignancy were excluded from the study. The collected PAP smears and histological samples were analyzed by a board certified pathologist specialized in Gynaecological Oncology. The study was conducted according to the Declaration of Helsinki.

The Institutional Review Board at the Medical University of Vienna approved this study (Reference: EK number 017/2004).

2.1. DNA Isolation. DNA was extracted from patients' cervical smear using QIAamp DNA Blood Mini Kit (QIAGEN, Germany) according to the instruction of manufacturer. DNA was stored at $4^{\circ} \mathrm{C}$ until the samples were further processed.

DNA was amplified with polymerase chain reaction (PCR) and DNA pyrosequencing was performed according to established protocols as described previously [23]. After researching $d b S N P$, the SNP database from National Center for Biotechnology Information, and PubMed, the five most common and clinically relevant TAP gene polymorphisms (i.e., TAP 1341, TAP 2254, TAP 1135, TAP 1693, and TAP 1993) were chosen for investigation.

2.2. TAP Genotyping. Genotyping the five polymorphisms was carried out using pyrosequencing. Primers used for the amplification of the fragments including the polymorphic sites, the length of the PCR products, and the sequencing primers were shown in Table 1. PCR was carried out in a total volume of $25 \mu \mathrm{L}$ including $25 \mathrm{ng}$ total DNA, 5 pmol of each sense and antisense primers and $12.5 \mu \mathrm{L}$ JumpStart REDTaq ReadyMix Reaction Mix (SIGMA, Missouri, USA), which contains $20 \mathrm{mM}$ Tris- $\mathrm{HCl}, \mathrm{pH} 8.3,100 \mathrm{mM} \mathrm{KCl}, 4 \mathrm{~m} \mathrm{MgCl}_{2}$, $0.002 \%$ gelatin, $0.4 \mathrm{mM}$ each $\mathrm{dNTP}$, inert dye, stabilizers, 0.06 unit/ $\mu \mathrm{L}$ Taq DNA Polymerase, JumpStart Taq antibody. The reaction was performed on a Perkin-Elmer GeneAmp PCR system 9600 with 40 cycles at $94^{\circ} \mathrm{C}$ for 30 seconds, at the corresponding annealing temperature (see Table 1) for 30 seconds and $72^{\circ} \mathrm{C}$ for 30 seconds. The reaction was preceded by a primary denaturation step at $94^{\circ} \mathrm{C}$ for 1 minute and incubated at $72^{\circ} \mathrm{C}$ for $7 \mathrm{~min}$ at last.

Polymorphisms were detected using Pyrosequencer PSQ 96 and PSQ 96 SNP Reagent Kit. $25 \mu \mathrm{L}$ PCR product was used for pyrosequencing according to the instruction of the manufactory. $5 \mathrm{pmol}$ of the sequencing primer was applied to detect the polymorphisms.

2.3. Statistical Analysis. Hardy-Weinberg equilibrium was tested by chi-square tests comparing observed and expected genotype frequencies. Differences in gene polymorphism frequencies and smoking between women with CIN and controls were assessed by chi-square tests; results are given as $P$ value and odds ratio (OR) (95\% confidence interval $(95 \% \mathrm{CI}))$. In a multivariate logistic regression model, we 
TABLE 1: Analysis of the five TAP gene polymorphisms.

\begin{tabular}{|c|c|c|c|c|}
\hline SNP & Allele specific probes & PCR product & Sequencing primer & $\begin{array}{l}\text { Annealing } \\
\text { temperature }\end{array}$ \\
\hline TAP1 1341 & $\begin{array}{l}\text { biotin-5'-TACCTGGTGCGAGGCCTATGTC } \\
5^{\prime} \text {-AGCGGGCCAACTCCATGAAC }\end{array}$ & $150 \mathrm{bp}$ & $5^{\prime}$-AAAAGCAGAGGCAGG & $64^{\circ} \mathrm{C}$ \\
\hline TAP1 2254 & $\begin{array}{l}5^{\prime} \text {-CATGTGGCTATACCGTTC } \\
\text { biotin-5'-GGATAAGTACACACGGTTTC }\end{array}$ & $135 \mathrm{bp}$ & $5^{\prime}$-TTGCTCTGCAGAGGTAG-3’ & $52^{\circ} \mathrm{C}$ \\
\hline TAP2 1135 & $\begin{array}{l}5^{\prime} \text {-GAAGTCTGTCGCTATAAAGAG } \\
\text { biotin-5'-GGCTCCTTTCACAACCAC }\end{array}$ & $124 \mathrm{bp}$ & $5^{\prime}$-CGCCTTGTACCTGCT-3' & $52^{\circ} \mathrm{C}$ \\
\hline TAP2 1693 & $\begin{array}{l}\text { biotin-5'-GCACAGGTGGTTTCAGTTGG } \\
5^{\prime} \text {-CTTATCATCTTCGCAGCTCTGC }\end{array}$ & $96 \mathrm{bp}$ & $5^{\prime}$-GCTCTGCAGCCCATA-3' & $58^{\circ} \mathrm{C}$ \\
\hline TAP2 1993 & $\begin{array}{l}5^{\prime} \text {-TCTTTCTGAGGCACTGTGATC } \\
\text { biotin-5' -GGAAACTCAAAGCAGGAAC }\end{array}$ & $283 \mathrm{bp}$ & $5^{\prime}$-TGATTGCTCACAGGCT-3' & $54^{\circ} \mathrm{C}$ \\
\hline
\end{tabular}

SNP: single nucleotide polymorphism.

PCR: polymerase chain reaction.

TAP: transporter associated with antigen processing.

TABLe 2: Patients' characteristics broken down by women with cervical intraepithelial neoplasia (CIN) and controls.

\begin{tabular}{|c|c|c|c|c|}
\hline Characteristics & Controls & $\mathrm{CIN}$ & $P$ & OR $(95 \% \mathrm{CI})$ \\
\hline Total number & 206 & 616 & - & \\
\hline Histology & & & - & \\
\hline CIN 1 & - & $206(33.4 \%)$ & & \\
\hline CIN 2 & - & $205(33.3 \%)$ & & \\
\hline CIN 3 & - & $205(33.3 \%)$ & & \\
\hline Smoking & & & $0.0001^{\mathrm{a}}$ & $2.6(1.7-3.9)$ \\
\hline No & $70(64.2 \%)$ & $225(41.2 \%)$ & & \\
\hline Yes & $39(35.8 \%)$ & $321(58.8 \%)$ & & \\
\hline HPV high-risk infection & & & - & \\
\hline Negative & - & $61(14.5 \%)$ & & \\
\hline Positive & - & $361(85.5 \%)$ & & \\
\hline
\end{tabular}

${ }^{a}$ Statistical analysis by chi-square test.

CIN: cervical intraepithelial neoplasia.

HPV: human papillomavirus.

OR ( $95 \% \mathrm{CI})$ : odds ratio ( $95 \%$ confidence interval).

assessed the independent association between TAP gene polymorphisms, smoking, and risk of CIN. Differences in gene polymorphism frequencies between women with CIN 1 and CIN 2/3 were assessed by chi-square tests; results are given as $P$ value and odds ratio (OR) (95\% confidence interval $(95 \% \mathrm{CI}))$.

We used the software SAS System (Version 9.1 SAS Institute Inc., Cary, NC, USA) and SPSS (SPSS 19.0, SPSS Inc. Chicago, IL, USA) for statistical analysis. Two-sided $P$ values $\leq 0.05$ were considered statistically significant.

Haplotypes can only be estimated and then probability values between 0 and 1 are assigned, expressing the likelihood for each haplotype to be present in one allele of a patient. These probabilities sum up to 2 in each patient (for the two alleles) and were included as continuous covariates (one for each haplotype) in the logistic regression model. Haplotypes occurring with a frequency $<5.0 \%$ were pooled. A logistic regression model was estimated to evaluate whether the presence of one or more copies of each particular haplotype in a patient is associated with a higher or lower risk of CIN, compared to a patient with two copies of the wildtype haplotype. Haplotype analysis was performed by using SAS/Genetics software.

\section{Results}

Women with CIN presented with the following histological results: CIN I $n=206 / 616$ (33.4\%), CIN II $n=205 / 616$ (33.3\%), and CIN III $n=205 / 616$ (33.3\%). Sixty-one of 422 (14.5\%) women with CIN were tested negative and 361 of 422 (85.5\%) positive for high-risk HPV infection. Of note, in 191 of $616(46.0 \%)$ patients HPV tests are missing. Patients with CIN (58.8\%) were smoking significantly more often than controls (35.8\%) $(P=0.0001)$ (Table 2).

The TAP SNPs 1341, 2254, 1135, 1693, and 1993 were genotyped in 616 patients with CIN and 206 controls. The genotype frequencies for all the studied SNP's were in HardyWeinberg equilibrium in both controls and patients with 
TABLE 3: Genotype and allele frequencies of the five investigated TAP gene polymorphisms in women with cervical intraepithelial neoplasia $(\mathrm{CIN})$ and controls.

\begin{tabular}{|c|c|c|c|c|}
\hline Gene polymorphisms & Controls (\%) & CIN (\%) & $P^{\mathrm{a}}$ & OR $(95 \% \mathrm{CI})$ \\
\hline TAP 1341 & & & 0.08 & $1.4(1.0-2.0)$ \\
\hline wt/wt & $155(75.2 \%)$ & $423(68.7 \%)$ & & \\
\hline $\mathrm{wt} / \mathrm{mt}$ & $44(21.4 \%)$ & $172(27.9 \%)$ & & \\
\hline $\mathrm{mt} / \mathrm{mt}$ & $7(3.4 \%)$ & $21(3.4 \%)$ & & \\
\hline TAP 2254 & & & 0.3 & $1.3(0.9-1.8)$ \\
\hline wt/wt & $161(78.2 \%)$ & $455(73.9 \%)$ & & \\
\hline $\mathrm{wt} / \mathrm{mt}$ & $39(18.9 \%)$ & $148(24.0 \%)$ & & \\
\hline $\mathrm{mt} / \mathrm{mt}$ & $6(2.9 \%)$ & $12(1.9 \%)$ & & \\
\hline TAP 1135 & & & 0.8 & $0.9(0.7-1.3)$ \\
\hline $\mathrm{wt} / \mathrm{wt}$ & $143(69.4 \%)$ & $435(70.6 \%)$ & & \\
\hline $\mathrm{wt} / \mathrm{mt}$ & $51(24.8 \%)$ & $165(26.8 \%)$ & & \\
\hline $\mathrm{mt} / \mathrm{mt}$ & $12(5.8 \%)$ & $16(2.6 \%)$ & & \\
\hline TAP 1693 & & & 0.3 & $1.3(0.8-2.2)$ \\
\hline wt/wt & $184(89.3 \%)$ & $531(86.2 \%)$ & & \\
\hline $\mathrm{wt} / \mathrm{mt}$ & $22(10.7 \%)$ & $84(13.6 \%)$ & & \\
\hline $\mathrm{mt} / \mathrm{mt}$ & $0(0 \%)$ & $1(0.2 \%)$ & & \\
\hline TAP 1993 & & & 0.2 & $0.8(0.6-1.1)$ \\
\hline wt/wt & $90(43.7 \%)$ & $301(48.9 \%)$ & & \\
\hline $\mathrm{wt} / \mathrm{mt}$ & $92(44.7 \%)$ & $248(40.3 \%)$ & & \\
\hline $\mathrm{mt} / \mathrm{mt}$ & $24(11.7 \%)$ & $66(10.7 \%)$ & & \\
\hline
\end{tabular}

${ }^{a}$ Statistical analysis by chi-square test are calculated $\mathrm{wt} / \mathrm{wt}$ versus $\mathrm{wt} / \mathrm{mt}$ and $\mathrm{mt} / \mathrm{mt}$.

TAP: transporter associated with antigen processing.

CIN: cervical intraepithelial neoplasia.

OR (95\% CI): odds ratio (95\% confidence interval).

TABLE 4: Multivariable regression model to determine independent risk factors for cervical intraepithelial neoplasia (CIN).

\begin{tabular}{lcc}
\hline & \multicolumn{2}{c}{ Multivariable regression } \\
& $P$ & OR (95\% CI) \\
\hline $\begin{array}{l}\text { TAP 1341 } \\
\text { (wt/wt versus wt/mt and mt/mt) }\end{array}$ & 0.07 & $2.5(0.9-7.0)$ \\
$\begin{array}{l}\text { TAP 2254 } \\
\text { (wt/wt versus wt/mt and mt/mt) }\end{array}$ & 0.3 & $0.6(0.2-1.6)$ \\
$\begin{array}{l}\text { TAP } 1135 \\
\text { (wt/wt versus wt/mt and } \mathrm{mt} / \mathrm{mt})\end{array}$ & 0.8 & $1.1(0.6-1.8)$ \\
$\begin{array}{l}\text { TAP } 1693 \\
\text { (wt/wt versus wt/mt and } \mathrm{mt} / \mathrm{mt})\end{array}$ & 0.5 & $0.8(0.4-1.6)$ \\
$\begin{array}{l}\text { TAP 1993 } \\
\text { (wt/wt versus wt/mt and mt/mt) }\end{array}$ & 0.7 & $0.9(0.6-1.4)$ \\
$\begin{array}{l}\text { Smoking } \\
\text { (no versus yes) }\end{array}$ & 0.002 & $1.9(1.3-2.9)$ \\
\hline
\end{tabular}

TAP: transporter associated with antigen processing.

CIN: cervical intraepithelial neoplasia.

OR (95\% CI): odds ratio ( $95 \%$ confidence interval).

CIN. Genotype frequencies of the five investigated TAP gene polymorphisms are given in Table 4. Distribution of genotypes for the five SNP's did not differ significantly between patients with CIN and controls (Table 3). In a multivariate model, smoking, but none of the investigated five SNPs, was independently associated with risk of CIN (Table 4).

Moreover, in 616 patients with CIN the risk of high-grade CIN was investigated. No significant association was found between the investigated TAP genotypes and risk for highgrade CIN $(P=0.08$ for TAP 1341; $P=0.3$ for TAP 2254; $P=0.8$ for TAP $1135 ; P=0.3$ for TAP $1693 ; P=0.2$ for TAP 1993) (Table 3).

The combined effect of the five TAP gene polymorphisms on the risk for cervical intraepithelial neoplasia was investigated by haplotype analysis (Table 5). Haplotype analysis revealed that women with haplotype mut-wt-wt-wtwt (TAP polymorphisms t1135-t1341-t1693-t1993-t2254) had a significantly lower risk for CIN, compared to women with the haplotype wt-wt-wt-wt-wt $(P=0.006$; OR $0.5[0.35-0.84])$.

\section{Discussion}

The aim of the present study was to evaluate the association between five common TAP gene polymorphisms and risk for CIN. The haplotype combination mut-wt-wt-wtwt (TAP polymorphisms t1135-t1341-t1693-t1993-t2254) was associated with a reduced risk for the presence of cervical intraepithelial neoplasia (OR $0.5(0.4-0.8)$ ).

This finding seems biologically plausible, as TAP facilitates the detection of HPV by MHC-I molecules and contributes to successful detection and eradication of HPV 
TABLE 5: Association between TAP gene haplotype combinations and risk of cervical intraepithelial neoplasia (CIN).

\begin{tabular}{lcccc}
\hline \multirow{2}{*}{ Haplotypes } & \multicolumn{2}{c}{ Haplotype frequency } & \multirow{2}{*}{ OR (95\% CI) } \\
\hline (t1135-t1341-t1693-t1993-t2254) & Controls in \% & & \\
wt-wt-wt-wt-wt (reference) & & $28.8 \%$ & - & 1.0 \\
mut-wt-wt-wt-wt & $31.2 \%$ & $7.3 \%$ & 0.006 & $0.5(0.4-0.8)$ \\
wt-mut-wt-wt-mut & $9.7 \%$ & $6.5 \%$ & 0.7 & $0.1(0.6-1.9)$ \\
wt-wt-wt-mut-wt & $5.5 \%$ & $16.4 \%$ & $0.8(0.6-1.1)$ \\
Others $^{\mathrm{a}}$ & $20.9 \%$ & $43.6 \%$ & 0.1 & $2.0(0.8-4.7)$ \\
\hline
\end{tabular}

${ }^{\mathrm{a}}$ Haplotypes $<5 \%$ were pooled ( $=$ others).

TAP: transporter associated with antigen processing.

CIN: cervical intraepithelial neoplasia.

OR (95\% CI): odds ratio ( $95 \%$ confidence interval).

despite various immunoevasion mechanisms of the virus [2]. Although the biological implication of the observed haplotype combination is not fully elucidated, this haplotype combination might facilitate the detection of HPV and subsequently reduce the risk for CIN development.

Our observation is in line with studies investigating the role of TAP gene polymorphisms and risk for high-grade $\mathrm{CIN}$ and cervical cancer $[7,13]$. Einstein et al. ascertained a reduced risk for high-grade CIN at the presence of TAP1 I333V $(P=0.02)$ and TAP1 D637G $(P=0.01)$. In women with cervical cancer, a TAP2 gene polymorphism has been associated with a reduced risk for cervical cancer [13]. Of note, this study was performed in an Indian cohort although no population diversities for these gene polymorphisms are described.

Interestingly, we were not able to ascertain any association between five common TAP polymorphisms and risk of CIN in a relatively large number of women. As we did not aim to evaluate the exact biological mechanisms exerted by the five TAP gene polymorphisms or their haplotype combinations, we did not investigate MHC-I serum levels and we did not obtain tissue of the patients. Moreover, patients in the present study represent a highly selected group of women, as all patients were of Caucasian origin and have been recruited at a single tertiary care center in Vienna, Austria.

In a multivariate model, smoking, but none of the investigated five SNPs, was independently associated with risk of CIN. It is well known that women smoking cigarettes are at higher risk for development of CIN. Due to these facts patients should be advised to quit smoking to reduce the risk for high-grade CIN development and increase spontaneous regression.

In the present study the protective effect of a TAP1 and TAP2 haplotype combination for the presence of CIN has been observed in a relatively large cohort of Caucasian women. Although its further implication has to be elucidated, this haplotype combination could be used to identify women, less susceptible for development of CIN following an infection with high-risk human papillomavirus.

\section{Conflict of Interests}

The authors declare that they have no conflict of interests.

\section{Acknowledgments}

The authors thank Eva Wolf and Andrea Schuster for their critical help at storing and analysing the study samples.

\section{References}

[1] M. R. McCredie, K. J. Sharples, C. Paul et al., "Natural history of cervical neoplasia and risk of invasive cancer in women with cervical intraepithelial neoplasia 3: a retrospective cohort study," The Lancet Oncology, vol. 9, no. 5, pp. 425-434, 2008.

[2] D. M. Parkin and F. Bray, "Chapter 2: the burden of HPV-related cancers," Vaccine, vol. 24, no. 3, pp. S11-S25, 2006.

[3] M. H. Schiffman, H. M. Bauer, R. N. Hoover et al., "Epidemiologic evidence showing that human papillomavirus infection causes most cervical intraepithelial neoplasia," Journal of the National Cancer Institute, vol. 85, no. 12, pp. 958-964, 1993.

[4] E. M. de Villiers, D. Wagner, A. Schneider et al., "Human papillomavirus infections in women with and without abnormal cervical cytology," The Lancet, vol. 2, no. 8561, pp. 703-705, 1987.

[5] M. H. Schiffman, "New epidemiology of human papillomavirus infection and cervical neoplasia," Journal of the National Cancer Institute, vol. 87, no. 18, pp. 1345-1347, 1995.

[6] S. Collins, T. P. Rollason, L. S. Young, and C. B. J. Woodman, "Cigarette smoking is an independent risk factor for cervical intraepithelial neoplasia in young women: a longitudinal study," European Journal of Cancer, vol. 46, no. 2, pp. 405-411, 2010.

[7] P. Appleby, V. Beral, A. Berrington de González et al., "Carcinoma of the cervix and tobacco smoking: collaborative reanalysis of individual data on 13,541 women with carcinoma of the cervix and 23,017 women without carcinoma of the cervix from 23 epidemiological studies," International Journal of Cancer, vol. 118, no. 6, pp. 1481-1495, 2006.

[8] F. V. Cromme, J. Airey, M.-T. Heemels et al., "Loss of transporter protein, encoded by the TAP-1 gene, is highly correlated with loss of HLA expression in cervical carcinomas," Journal of Experimental Medicine, vol. 179, no. 1, pp. 335-340, 1994.

[9] M. E. Connor and P. L. Stern, "Loss of MHC class-I expression in cervical carcinomas," International Journal of Cancer, vol. 46, no. 6, pp. 1029-1034, 1990.

[10] T. Spies, M. Bresnahan, S. Bahram et al., "A gene in the human major histocompatibility complex class II region controlling the class I antigen presentation pathway," Journal of Immunology, vol. 180 , no. 5 , pp. 2737-2740, 2008. 
[11] R. Abele and R. Tampé, "Function of the transport complex TAP in cellular immune recognition," Biochimica et Biophysica Acta, vol. 1461, no. 2, pp. 405-419, 1999.

[12] D. Parcej and R. Tampé, "ABC proteins in antigen translocation and viral inhibition," Nature Chemical Biology, vol. 6, no. 8, pp. 572-580, 2010.

[13] P. Cresswell, N. Bangia, T. Dick, and G. Diedrich, "The nature of the MHC class I peptide loading complex," Immunological Reviews, vol. 172, pp. 21-28, 1999.

[14] R. N. Germain, "MHC-dependent antigen processing and peptide presentation: providing ligands for T lymphocyte activation," Cell, vol. 76, no. 2, pp. 287-299, 1994.

[15] M.-T. Heemels and H. Ploegh, "Generation, translocation, and presentation of MHC class I-restricted peptides," Annual Review of Biochemistry, vol. 64, pp. 463-491, 1995.

[16] P. Thor Straten, A. F. Kirkin, T. Seremet, and J. Zeuthen, "Expression of transporter associated with antigen processing 1 and 2 (TAP1/2) in malignant melanoma cell lines," International Journal of Cancer, vol. 70, pp. 582-586, 1997.

[17] F. V. Cromme, C. J. L. M. Meijer, P. J. F. Snijders et al., "Analysis of MHC class I and II expression in relation to presence of HPV genotypes in premalignant and malignant cervical lesions," British Journal of Cancer, vol. 67, no. 6, pp. 1372-1380, 1993.

[18] V. Cerundolo, J. Alexander, K. Anderson et al., "Presentation of viral antigen controlled by a gene in the major histocompatibility complex," Nature, vol. 345, no. 6274, pp. 449-452, 1990.

[19] M. H. Einstein, S. Leanza, L. G. Chiu et al., "Genetic variants in TAP are associated with high-grade cervical neoplasia," Clinical Cancer Research, vol. 15, no. 3, pp. 1019-1023, 2009.

[20] D. M. Kordi Tamandani, R. C. Sobti, M. Shekari, S. A. Husseini, and V. Suri, "No association of TAP1 and TAP2 genes polymorphism with risk of cervical cancer in north Indian population," Journal of Assisted Reproduction and Genetics, vol. 26, no. 4, pp. 173-178, 2009.

[21] N. L. Fowler and I. H. Frazer, "Mutations in TAP genes are common in cervical carcinomas," Gynecologic Oncology, vol. 92, no. 3, pp. 914-921, 2004.

[22] B. S. Gostout, G. A. Poland, E. S. Calhoun et al., "TAP1, TAP2, and HLA-DR2 alleles are predictors of cervical cancer risk," Gynecologic Oncology, vol. 88, no. 3, pp. 326-332, 2003.

[23] C. Grimm, R. Watrowski, K. Baumühlner et al., "Genetic variations of interleukin-1 and -6 genes and risk of cervical intraepithelial neoplasia," Gynecologic Oncology, vol. 121, no. 3, pp. 537-541, 2011. 


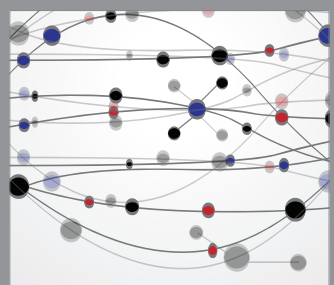

The Scientific World Journal
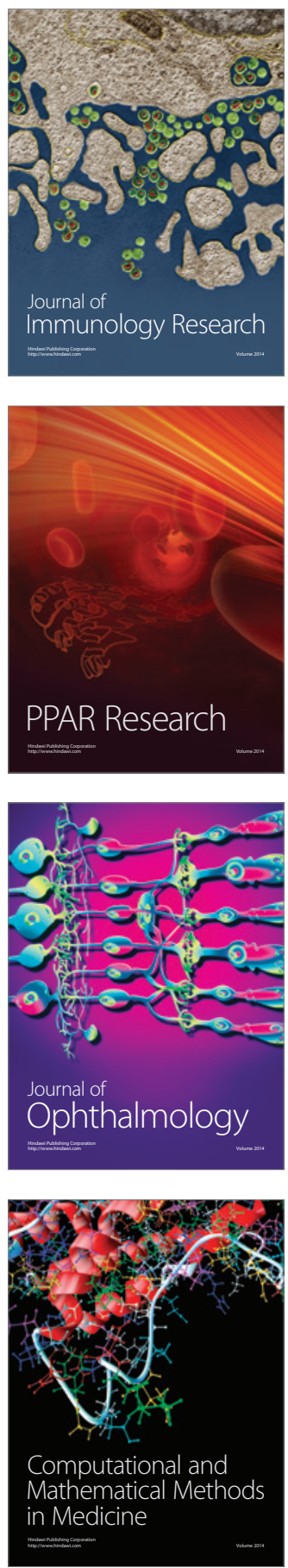

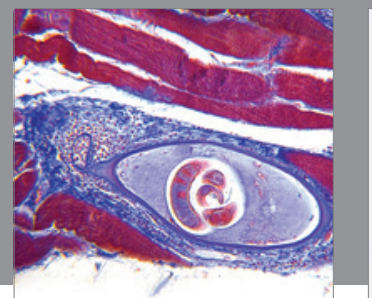

Gastroenterology

Research and Practice
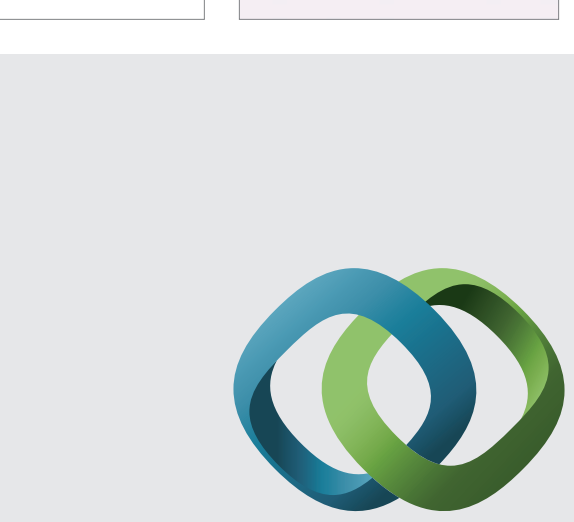

\section{Hindawi}

Submit your manuscripts at

http://www.hindawi.com
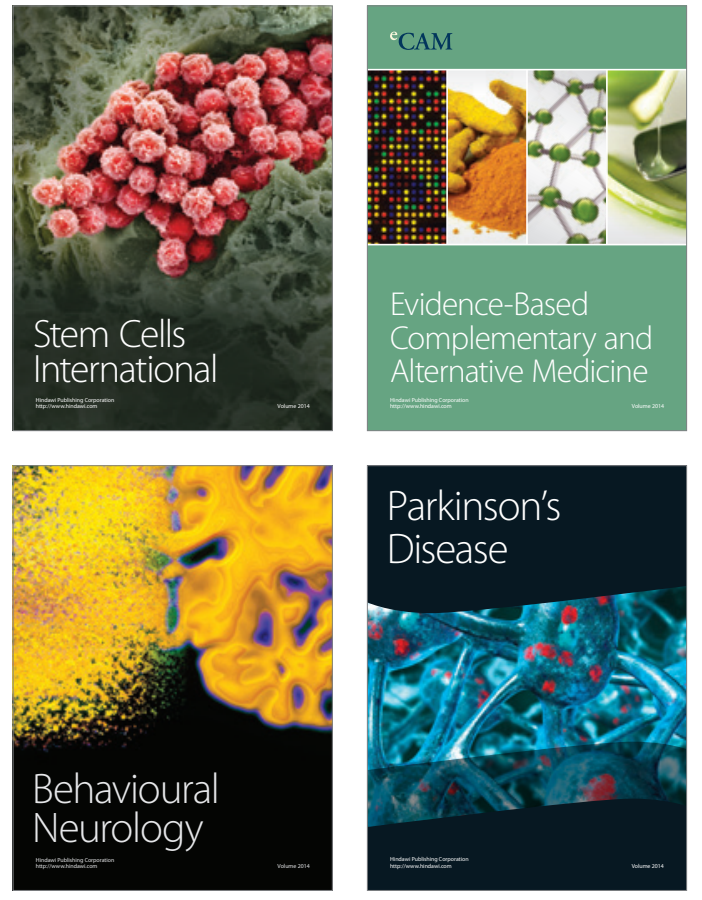
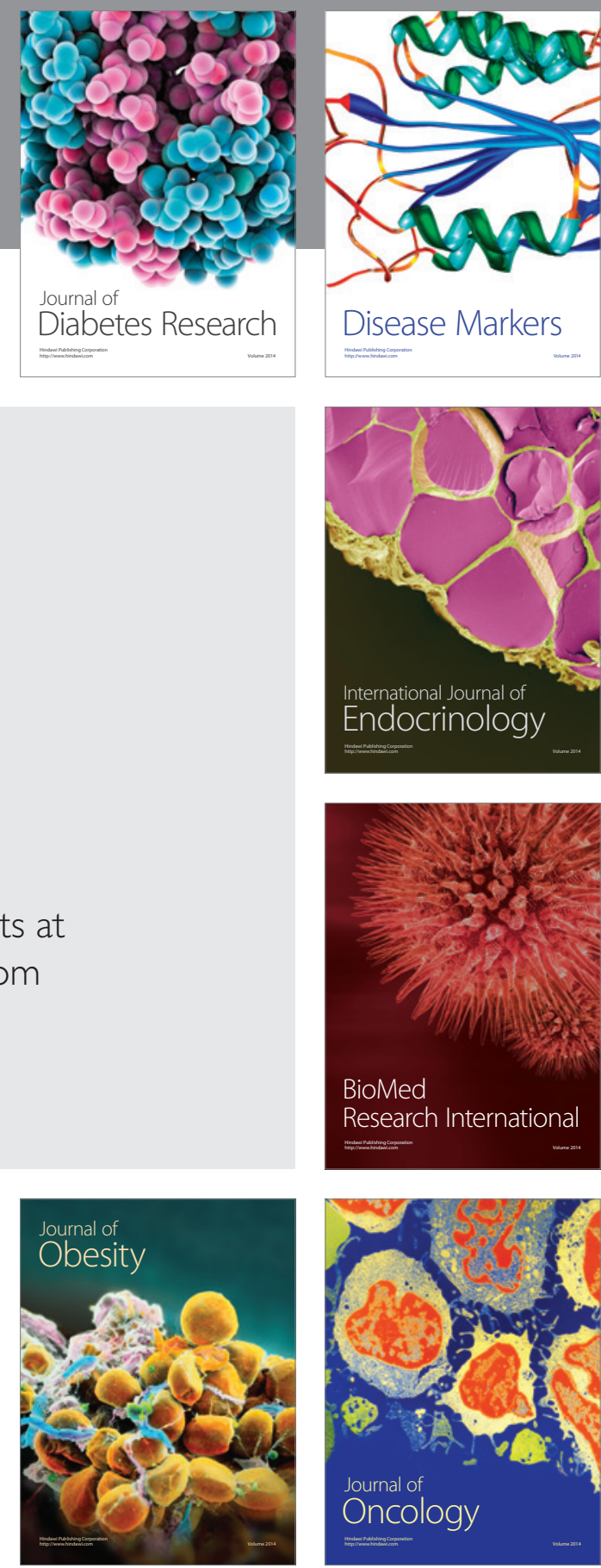

Disease Markers
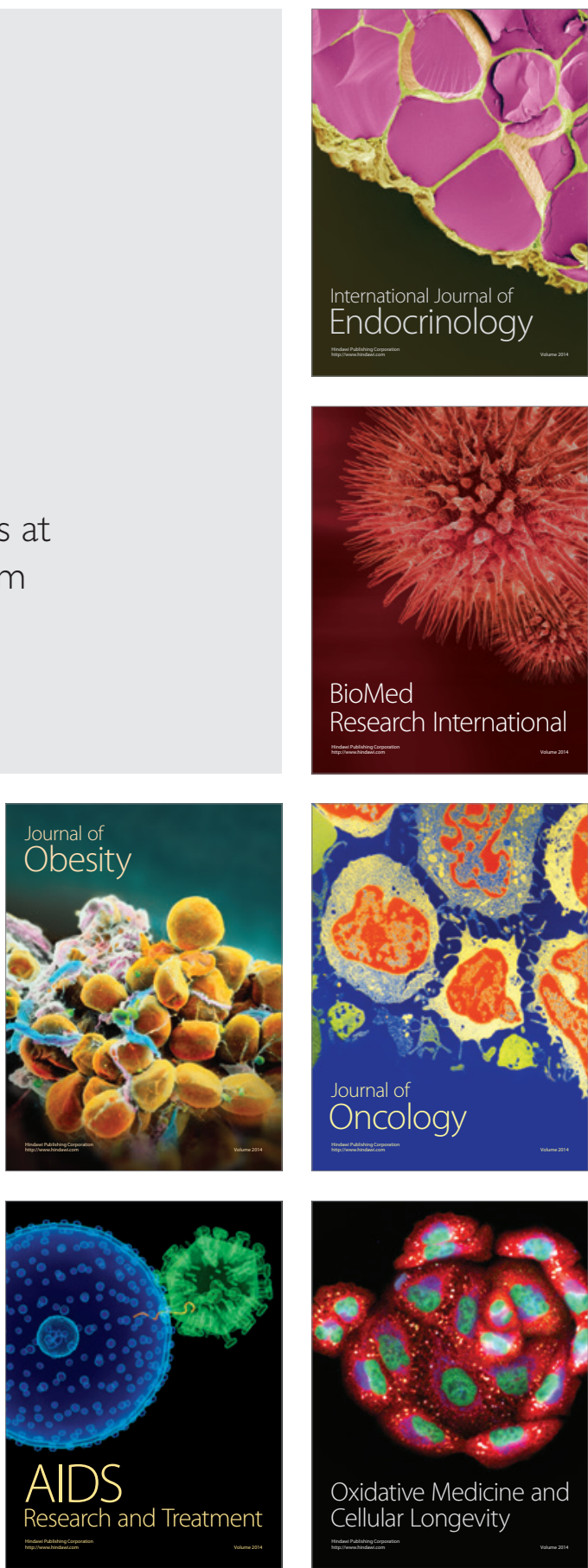